\title{
Performance analysis of the European X-ray Free Electron Laser 3.9 GHz superconducting cavities
}

\author{
M. Bertucci, A. Bignami, A. Bosotti, P. Michelato, L. Monaco, C. Pagani, \\ R. Paparella, and D. Sertore \\ INFN Sezione di Milano-Laboratorio LASA, Via Fratelli Cervi 201, 20090 Segrate (Mi), Italy \\ C. Maiano and P. Pierini \\ European Spallation Source ERIC, 22484 Lund, Sweden \\ J. Chen \\ Shanghai Institute of Applied Physics, Chinese Academy of Sciences, Shanghai 201800, China
}

(Received 3 January 2019; published 12 August 2019)

\begin{abstract}
The limits of performance of the European XFEL $3.9 \mathrm{GHz}$ superconducting cavities were investigated. Most cavities exhibited high field Q slope, reaching the breakdown field at approximately $22 \mathrm{MV} / \mathrm{m}$. We hypothesize that this limit is a feature of high frequency cavities and can be explained by a thermal model incorporating field dependent surface resistance. The results obtained from simulations were in good agreement with experimental data obtained at $2 \mathrm{~K}$.
\end{abstract}

DOI: 10.1103/PhysRevAccelBeams.22.082002

\section{INTRODUCTION}

Twenty third-harmonic $3.9 \mathrm{GHz}$ superconducting cavities were fabricated and tested at LASA as part of the contribution of INFN and DESY to the European XFEL project (EXFEL). The third harmonic section of the XFEL injector serves to compensate the nonlinear distortion of longitudinal phasespace produced by the first acceleration stage. As a result the downstream bunch compression stage can deliver a higher current beam to the undulator section with improved transverse emittance. Two third-harmonic modules, each made of eight $3.9 \mathrm{GHz}$ cavities, have already been assembled and one is currently in operation in the injector section of the machine.

In previous work, the cavity design, the fabrication and the qualification testing at LASA have been discussed [1]. In addition, there was a preliminary analysis of vertical test results and a first qualitative interpretation of the mechanism limiting the cavity performance at high fields [2]. Now, a more in-depth analysis is presented. The theoretical modeling of $Q_{0}$ vs $E_{\text {acc }}$ is treated in detail and the quantitative predictions of this model are compared with the experimental results and data collected from cavity diagnostics.

\footnotetext{
*Also at Università degli Studi di Milano, Via Celoria 13, 20129 Milano, Italy.

Published by the American Physical Society under the terms of the Creative Commons Attribution 4.0 International license. Further distribution of this work must maintain attribution to the author(s) and the published article's title, journal citation, and DOI.
}

\section{FABRICATION AND TESTING OF THE 3.9 GHZ SERIES PRODUCTION CAVITIES}

\section{A. Production}

Experience from $3.9 \mathrm{GHz}$ series production has been described in detail in a previous paper [1]. We report here briefly only the main aspects involving the preparation of the rf surface. Due to their high frequency, 3.9 GHz cavities have a strong sensitivity to geometric variations arising from the fabrication and surface preparation process. Optimization and stabilization of the procedures have been achieved thanks to the previous experience of FNAL and DESY [3] during the development of the FLASH thirdharmonic system (ACC39). The production of three preseries cavities allowed the optimization of the process using the infrastructure provided by the qualified industrial vendor. The niobium material used in these cavities was supplied by Ningxia OTIC in two different batches of 60 niobium sheets. Both batches met the required material specifications of $R R R>300$, predominant grain size of $\mathrm{ASTM}=6$ or finer and no grains larger than ASTM $=6$. Every sheet $(2.8 \mathrm{~mm} \times 265 \mathrm{~mm} \times 265 \mathrm{~mm})$ was qualitychecked at DESY by the Eddy Current Scanning technique.

After equatorial welding of dumbbells, the cavity inner surface underwent the following treatment steps: (i) Bulk buffered chemical polishing (BCP) at a 1:1:2 volume ratio. Approx. $124 \mu \mathrm{m}$ of average thickness was removed in the first batch of 10 cavities. This was increased to $135 \mu \mathrm{m}$ for the second batch. A typical etching rate of $1 \mu \mathrm{m} / \mathrm{min}$ to $1.4 \mu \mathrm{m} / \mathrm{min}$ was achieved in all treatments. The acid mixture was chilled to ensure that cavity 
temperature was below $15^{\circ} \mathrm{C}$ during the treatment. (ii) External surface $\mathrm{BCP}$ at a $1: 1: 2$ volume ratio. Approx. $20 \mu \mathrm{m}$ of surface was removed. (iii) Annealing for 2 hours in an oven at $800^{\circ} \mathrm{C}$ to achieve hydrogen degassing. (iv) Final "Flash" BCP at a 1:1:2 volume ratio. Approximately $35 \mu \mathrm{m}$ of surface was removed for the first batch of 10 cavities. This was reduced to $20 \mu \mathrm{m}$ for the second batch. (v) High pressure rinse cycle. 12 hours at 100 bar with ultra pure water (UPW).

The bulk BCP process was performed in three steps to improve the uniformity of surface removal, reversing the cavity after each treatment. After each step, rinsing with UPW, weighing, drying, and frequency testing was performed in order to monitor the frequency response to the etching process. Two optical inspection processes-one immediately after electron beam welding and one after the $800{ }^{\circ} \mathrm{C}$ annealing-were performed to check for the presence of welding imperfections and large geometrical defects which could limit the cavity performance. These inspections revealed some suspect features close to welding seams in 7 cases, 6 after the EBW stage and one after the $800^{\circ} \mathrm{C}$ annealing. In the latter case (cavity $3 \mathrm{HZOO}$ ) the cavity underwent a further $15 \mathrm{~min}$ BCP treatment after grinding to remove the defect.

\section{B. Vertical acceptance testing as performed at the LASA facility}

The 20 series cavities were tested at the LASA vertical test facility to confirm the required EXFEL qualification values of $E_{\text {acc }}=15 \mathrm{MV} / \mathrm{m}$ and $Q_{0}=10^{9}$ at the operating temperature of $2 \mathrm{~K}$. The test cryostat allowed testing of two cavities at a time. The accelerating field was raised until a limiting mechanism prevented any further power rise. Second sound and fast thermometry sensors were employed for the detection of quench events. An external proportional counter was installed above the cryostat for measuring x-ray radiation due to field emission. A mumetal magnetic shield was installed, and a maximum value of $10 \mathrm{mG}$ was measured in the cavity region with a fluxgate triaxial magnetometer. A full description of the test facility, together with cavity preparation for the tests have been reported [1]. After the $2 \mathrm{~K}$ characterization, the helium bath temperature was lowered to $1.8 \mathrm{~K}$ or below and the power rise repeated.

The cavity surface resistance $R_{s}(T)=R_{\mathrm{BCS}}(T)+R_{0}$ was measured during the cool-down. SUPERFIT code [4] with the Halbritter quasiexponential formula for BCS surface resistance [5] was employed to separate the $R_{\mathrm{BCS}}(T)$ (BCS resistance) and $R_{0}$ (residual resistance) contributions. Reduced band gap $\frac{\Delta}{k_{B} T_{c}}$, electron mean free path $l_{e}$ and residual resistance $R_{0}$ were treated as free parameters in this fit, while $T_{c}=9.25 \mathrm{~K}, \lambda_{l}=32 \mathrm{~nm}$, and $\xi_{0}=39 \mathrm{~nm}$ were used as fixed parameters for critical temperature, London penetration depth and coherence length, respectively.

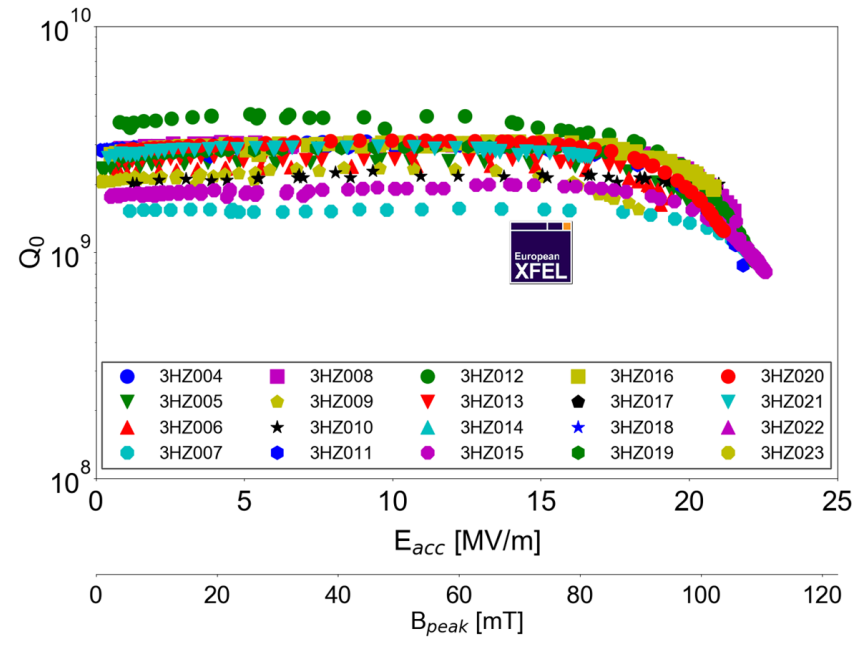

FIG. 1. Power rises for the 20 series production EXFEL $3.9 \mathrm{GHz}$ cavities at $2 \mathrm{~K}$. The qualification values $\left(E_{\text {acc }}=15 \mathrm{MV} / \mathrm{m}\right.$ and $Q_{0}=10^{9}$ ) are also shown.

The fit results have been reported previously [2]. In summary, a large spread was obtained for residual resistance, with values ranging between 14 and $85 \mathrm{n} \Omega$. With respect to BCS, the reduced band gap ranged between 1.78 and 1.90 and the electron mean free path ranged from $340 \AA$ (nearly the dirty limit) up to $2900 \AA$ (toward the clean limit). Assuming a first approximation $l_{e}=27 \cdot \mathrm{RRR}$ [6], this corresponded to a surface RRR in the 12-112 interval. Such a range of values, which is lower than the RRR bulk value of 300, may be justified by assuming a higher concentration of interstitials in the rf active layer. Experimental evidence of a near-surface hydrogen enriched layer with a thickness of about $100 \AA$ to $200 \AA$ has been reported following testing by the elastic recoil detection technique on niobium samples with treatment conditions similar to ours [7].

The power rises at $2 \mathrm{~K}$ for all the 20 cavities are shown in Fig. 1. A summary of cavity performance is outlined in Table I. Maximum accelerating field, $Q_{0}$ values at low $(1 \mathrm{MV} / \mathrm{m})$ and maximum accelerating field, and maximum external radiation (measured at the top of the cryostat) are shown.

All cavities except one met the EXFEL specifications, with the majority reaching $20 \mathrm{MV} / \mathrm{m}$, or more. There was a large spread in the low field $\mathrm{Q}$ values, due to the related spread in residual resistance $R_{0}$. With cavity $3 \mathrm{HZ} 022$, the observed anomalous value of $Q_{0}=4 \times 10^{9}$ is still under investigation. Regarding the performance at high fields, two different behaviors were observed: (i) Group I: Cavities 3HZ007, 3HZ010, 3HZ014, 3HZ019, 3HZ021, and 3HZ023 quenched all below $20 \mathrm{MV} / \mathrm{m}$, with an average maximum $E_{\text {acc }}$ of $16.6 \pm 1.5 \mathrm{MV} / \mathrm{m}$. The high field Q-value was almost equal to the one at low field. There was a clear change of maximum $E_{\text {acc }}$ with He bath temperature. Pointlike defects were detected by diagnostics. (ii) Group II: Cavities 3HZ004, 
TABLE I. Performances of $3.9 \mathrm{GHz}$ cavities.

\begin{tabular}{lcccc}
\hline \hline $\begin{array}{l}\text { Cavity } \\
\text { name }\end{array}$ & $\begin{array}{c}E_{\mathrm{acc}}^{\max } \\
{[\mathrm{MV} / \mathrm{m}]}\end{array}$ & $\begin{array}{c}Q_{0}\left(\times 10^{9}\right) \\
\text { at } 1 \mathrm{MV} / \mathrm{m}\end{array}$ & $\begin{array}{c}Q_{0}\left(\times 10^{9}\right) \\
\text { at } E_{\mathrm{acc}}^{\max }\end{array}$ & $\begin{array}{c}\text { ext. rad. } \\
{[\mu \mathrm{Sv} / \mathrm{h}]}\end{array}$ \\
\hline 3HZ004 & 20.0 & 2.03 & 0.99 & No \\
3HZ005 & 20.1 & 2.39 & 1.65 & No \\
3HZ006 & 20.9 & 2.30 & 2.02 & No \\
3HZ007 & 19.7 & 2.59 & 2.31 & No \\
3HZ008 & 21.0 & 2.39 & 1.51 & No \\
3HZ009 & 18.3 & 2.12 & 1.56 & No \\
3HZ010 & 18.2 & 1.56 & 1.55 & No \\
3HZ011 & 21.8 & 2.68 & 0.88 & 2 \\
3HZ012 & 20.8 & 2.69 & 2.12 & No \\
3HZ013 & 22.0 & 1.93 & 0.98 & No \\
3HZ014 & 15.0 & 2.91 & 2.89 & No \\
3HZ015 & 22.3 & 2.06 & 1.03 & No \\
3HZ016 & 21.1 & 2.74 & 1.90 & No \\
3HZ017 & 22.1 & 1.56 & 0.95 & No \\
3HZ018 & 21.8 & 2.63 & 1.46 & No \\
3HZ019 & 17.0 & 2.70 & 2.67 & No \\
3HZ020 & 21.0 & 2.72 & 1.26 & 0.2 \\
3HZ021 & 18.0 & 2.70 & 2.63 & No \\
3HZ022 & 22.1 & 4.11 & 0.93 & No \\
3HZ023 & 17.8 & 2.54 & 2.39 & No \\
\hline \hline
\end{tabular}

3HZ005, 3HZ008, 3HZ011, 3HZ012, 3HZ013, 3HZ015, 3HZ016, 3HZ017, 3HZ018, 3HZ020, and 3HZ022 quenched above $20 \mathrm{MV} / \mathrm{m}$, with an average maximum $E_{\text {acc }}$ of $21.3 \pm 0.8 \mathrm{MV} / \mathrm{m}$. All exhibit a high field Q slope, which begins at about $17 \mathrm{MV} / \mathrm{m}$. In some cases, this dramatically reduced the $\mathrm{Q}$ value to less than half of low field $Q_{0}$. There was almost no change in maximum $E_{\text {acc }}$ with He bath temperature. Cavity diagnostics did not identify any local quenches.

Outside the group I and group II behaviors were the cases of 3 HZ006 and 3HZ009. Only a modest reduction of $\mathrm{Q}$ occurred at the quench field of $20.9 \mathrm{MV} / \mathrm{m}$ in the former. A significant $\mathrm{Q}$ drop occurred, even at the lower quench field of $18.3 \mathrm{MV} / \mathrm{m}$, in the latter.

The difference of behavior is particularly evident when comparing the power rises at different temperatures. Figure 2 shows the power rises for cavity 3HZ021. An improvement of quench field for lower temperatures is evident, together with the increase in $Q_{0}$ value, as expected from BCS temperature dependence. A resistive defect, generating local thermal quench, is likely to be involved in the mechanism limiting cavity performance.

The power rises for cavity 3HZ015 at different bath temperatures are shown in Fig. 3. It is different from the previous case, with almost no gain in breakdown field at lower bath temperatures. The quench field at $1.7 \mathrm{~K}$ was only marginally higher than that at $2 \mathrm{~K}$. Even more surprising, the $\mathrm{Q}$ value at maximum field was essentially the same at all temperatures. Every Q vs $E_{\text {acc }}$ curve appears to converge in the same point, in spite of the difference in $\mathrm{BCS}$ resistance. In the insert, the power rise at $2 \mathrm{~K}$ is

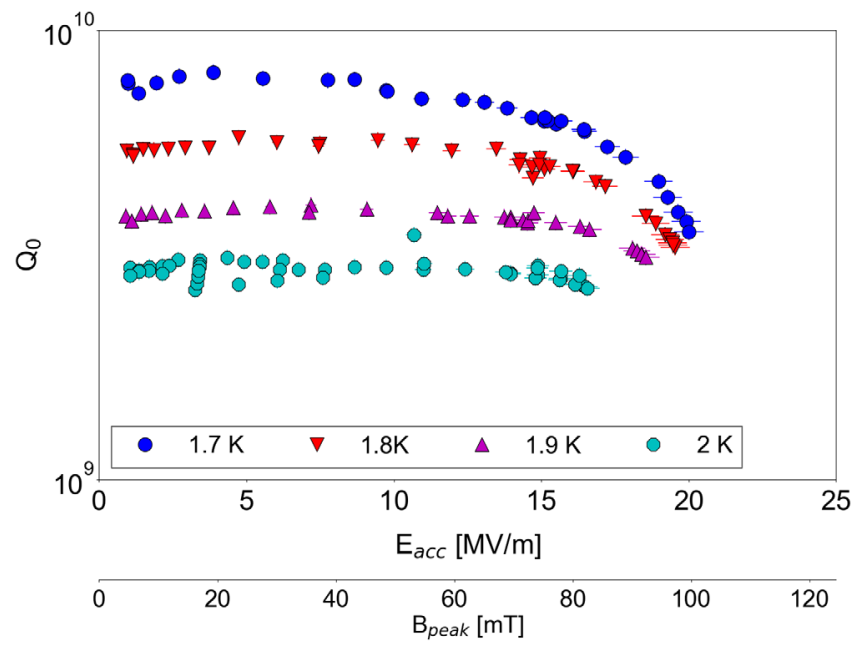

FIG. 2. Power rises for cavity $3 \mathrm{HZO21}$ at different $\mathrm{He}$ bath temperatures.

magnified in the medium field zone. As also noticed by experiments at FNAL [8], BCP treated $3.9 \mathrm{GHz}$ cavities exhibited a natural anti-Q slope at medium fields. In this case, we noticed a $10 \%$ increase of the initial value around $15 \mathrm{MV} / \mathrm{m}$, in line with the previously mentioned experimental results. On the whole, the high field behavior of cavity 3HZ015-and all others from the 2nd groupappeared to be linked to thermal breakdown. The simple model of a resistive defect based mechanism does not provide a good explanation, as there is essentially no gain in breakdown field when decreasing the bath temperature. Moreover, the reduction of Q-value suggest a global mechanism of dissipation which is triggered well before the breakdown field. Ultimately, the narrow range of breakdown fields-a few tenths of $\mathrm{MV} / \mathrm{m}$ around the average value of $21.3 \mathrm{MV} / \mathrm{m}$-led us to suspect an innate limiting mechanism in the $3.9 \mathrm{GHz}$ cavities prevailing at

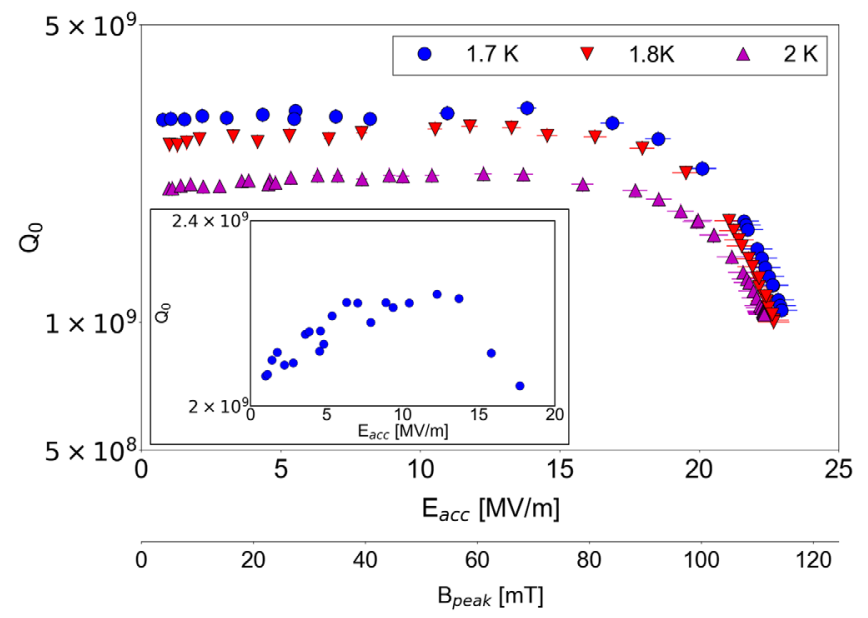

FIG. 3. Power rises for cavity $3 \mathrm{HZ} 015$. In the box, the Q vs $E_{\text {acc }}$ curve at $2 \mathrm{~K}$ is magnified in the $0-20 \mathrm{MV} / \mathrm{m}$ zone so to point out a slight medium field anti-Q slope. 
that field. The lower breakdown field observed with group I cavities could be due to true pointlike defects that, if removed, would result in cavity performances similar to those of group II. The next section, which deals with diagnostics of quench events, will supply new evidence for these views.

\section{Diagnostics for quench events}

The process of thermal breakdown on the cavity inner surface leads to a great dissipation of heat at the $\mathrm{Nb}-\mathrm{He}$ interface, synchronous with the drop of cavity transmitted power. This eventually increases the temperature of the surface and generates second sound shock waves in the superfluid helium. Both phenomena, if localized to a restricted area on the cavity surface, can be easily detected by means of fast thermometry and second sound sensors.

A thermometry sensor offers a means of directly identifying hot spot positions on the cavity surface but has a restricted spatial resolution. It is ineffective in detecting a quench if placed only few $\mathrm{cm}$ far away from the heat source. Therefore, an accurate thermometry mapping system needs to be employed incorporating several hundreds of sensors [9] in order to cover effectively the whole cavity active surface. Alternatively, a traditional second sound sensor-oscillating superleak transducer (OST) [10]—is able to detect the second sound waves coming from a wide area of the cavity surface, namely its whole line-of-sight. The simultaneous detection of the second sound signal by three sensors is therefore theoretically adequate to localize the quench position by means of trilateration.

In the case of $3.9 \mathrm{GHz}$ cavities, due to their small size, it is advantageous to apply the second sound diagnostic technique. Such a sensor is able to cover, by line of sight, a large portion of the cavity surface if placed appropriately. On the other hand, several error sources can affect the quench localization accuracy, such as the extended active sensor surface, the sometimes difficult identification of second sound pulse wave front and even additional phenomena altering thermodynamic properties of superfluid helium, producing local variations of second sound velocity [11]. As a consequence, spatial resolution of the quench zone is limited to $5 \mathrm{~mm}-10 \mathrm{~mm}$.

Given these considerations, we based our strategy for quench localization mainly on the second sound technique and less on fast thermometry, due to the limited number of available thermometry sensors. The visual inspection performed before testing gave an immediate indication of suspected points which could generate a thermal breakdown event. This provided the preferential sites for the placing of thermometry sensors. The second sound sensors were instead uniformly placed on the frame around the cavity, without any reference from optical inspection results. Thermometers (Cernox ${ }^{\circledR}$ and CCS $\left.{ }^{\circledR}\right)$ with fast readout electronics were attached to the cavity surface, with a four-wire configuration to read the resistance of the

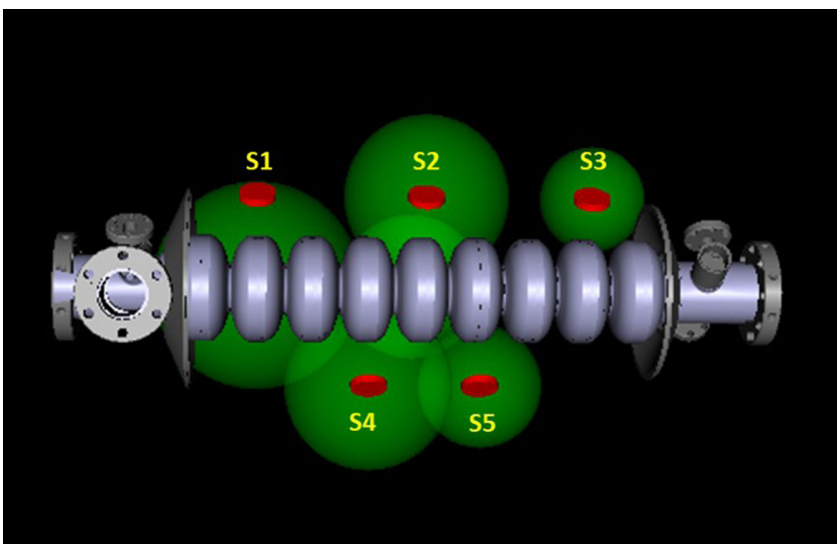

FIG. 4. Reconstruction of second sound signals for cavity 3HZ017. The spheres represent the quench detection range of the sensors.

sensor. In order to increase the reconstruction accuracy, 10 OST's were used for each cavity. Several algorithms of trilateration [12] were exploited for the reconstruction of the quench position.

Second sound signal analysis for cavities of group II (those quenching at high field with Q-slope) did not identify any isolated hot spots. Figure 4 shows a representative attempt to reconstruct a quench location for cavity $3 \mathrm{HZ} 017$ at $1.8 \mathrm{~K}$. The spheres represent all points with a distance $d_{i}=v(1.8 \mathrm{~K}) \cdot \Delta t_{i}$ from the sensor center, where $v(1.8 \mathrm{~K})$ is the second sound velocity in superfluid helium at $1.8 \mathrm{~K}$, and $\Delta t_{i}$ is the measured time of arrival of second sound wave-front at the $i$ th OST. For instance, sensor S3 points at cell 8 surface while S5 at cell 6, so it is unlikely that a single restricted zone on cavity surface would generate second sound waves.

Such behavior could be explained by assuming a global cavity heating mechanism as the trigger for second sound generation from high magnetic field zones near the cell equators. Conversely, group I cavities second sound signals are likely to come from a unique and well defined zone on the cavity surface.

Cavities 3HZ021 and 3HZ014 provided some interesting results. Cavity $3 \mathrm{HZ} 021$ prematurely quenched at $18 \mathrm{MV} / \mathrm{m}$, well below the average accelerating field exhibited by the series production, without any associated significant $Q$ value reduction. Figure 5 shows the results of the second sound quench reconstruction and the optical image of the quench area acquired after cavity welding. A shiny row, appearing just underneath the heat affected zone, is likely to be the source of cavity thermal breakdown. The defect was also present in the optical image taken after BCP treatment, confirming its role in the quench event.

Cavity 3HZ014 is the only one of the series production which did not meet, albeit marginally, the EXFEL specifications, with a quench event occurring at $15 \mathrm{MV} / \mathrm{m}$ without any reduction of Q-value. The result of second sound reconstruction is shown in Fig. 6 for a $1.8 \mathrm{~K}$ bath 


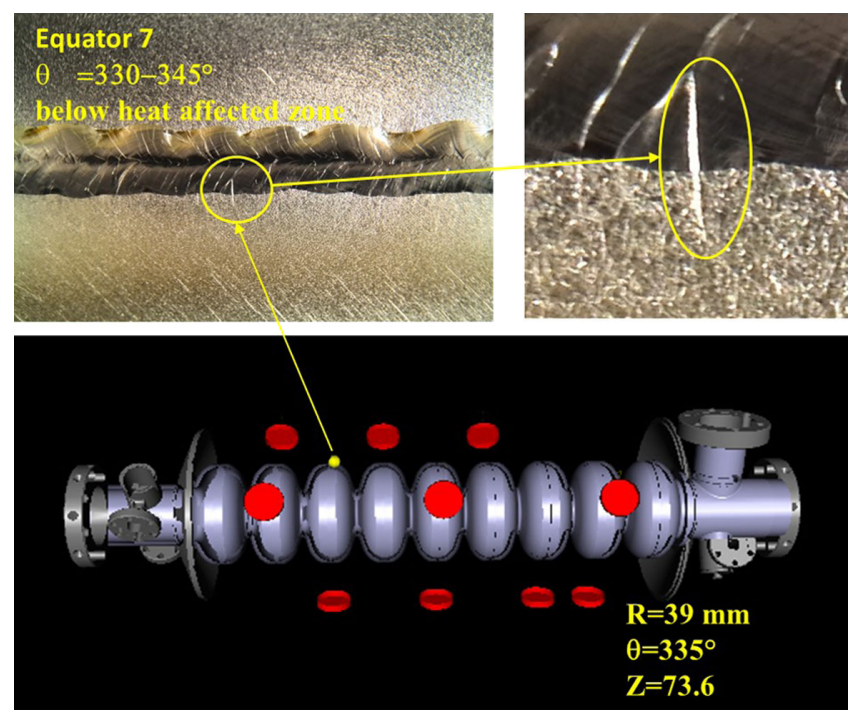

FIG. 5. Second sound quench reconstruction for cavity $3 \mathrm{HZO} 21$ (below) and optical image of the quench position (top left). On the top right, a magnification of the defect.

temperature, together with the corresponding optical image acquired in the inner cavity surface after $800{ }^{\circ} \mathrm{C}$ heat treatment. Optical inspection of this cavity after BCP treatment revealed several bumps on the equatorial weld zone. They were not visible in the image acquired after welding, indicating that this is a feature of the $\mathrm{BCP}$ treatment itself. Being aware that these defects could produce a premature cavity quench, the fast thermometry sensor C1 (Echo) was placed at the defect position to allow monitoring of the local temperature response during the cavity power rise. Figure 7 shows the time response of fast thermometry sensors together with the rf gate (pulsed mode with $25 \%$ duty cycle) and transmitted power.
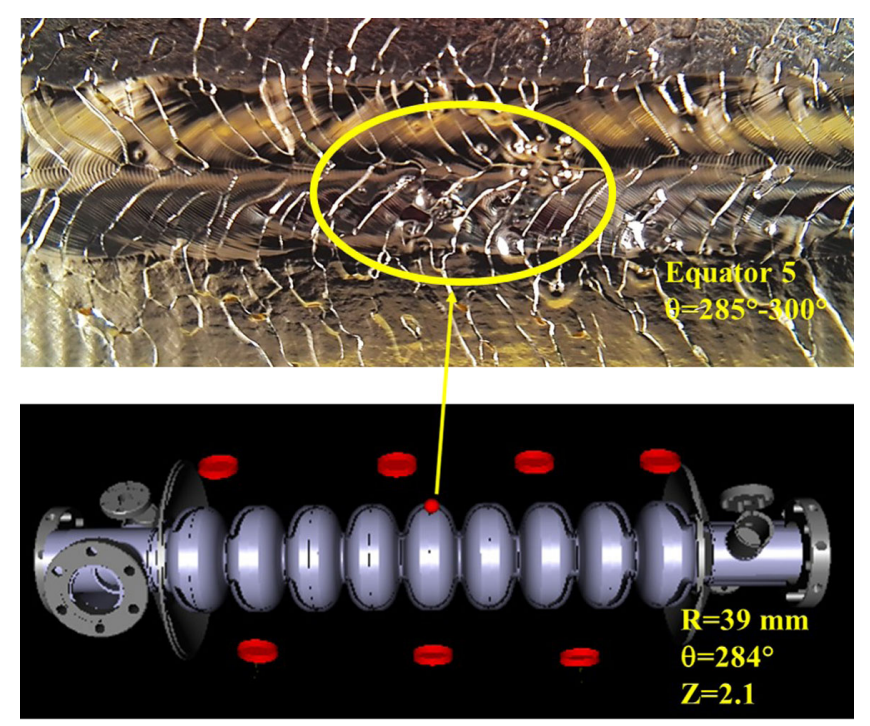

FIG. 6. Second sound quench reconstruction for cavity 3HZ014 (below) at $1.8 \mathrm{~K}$ and optical image of the quench position (top).

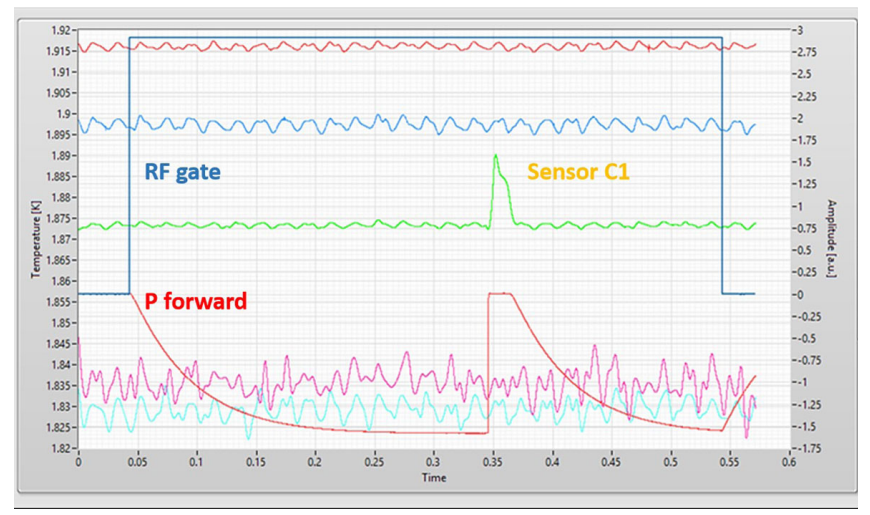

FIG. 7. Fast temperature sensors readout at the quench field. rf gate (violet) and transmitted power (red) and the sensor at the defect position (green) are highlighted.

Simultaneously with the drop of transmitted power (quench event), sensor C1 (Echo) registered a sudden temperature increase indicating a strong thermal transition on the cavity external surface, and an isolated hot spot generating second sound waves. This was associated with the premature thermal breakdown.

\section{MECHANISM OF PERFORMANCE LIMITATION}

\section{A. General considerations}

The quench behavior of the group I cavities at high field was different from that of group II. In the latter, a thermal breakdown was likely involved but there was no precise indication of a local hot spot. Several important clues were found from the analysis of power rises. There was an almost constant quench field in the interval 20-22 MV/m with an absence of any maximum gradient improvement with decreasing He bath temperature and a dramatic reduction of Q value while approaching the quench field. Putting this all together, a mechanism based on global thermal limitation can be suggested as the cause of gradient limitation for BCP treated $3.9 \mathrm{GHz}$ cavities. This is also supported by similar results obtained by FNAL [13] in the development of FLASH third harmonic system. Even in that case, the occurrence of cavity quench at similar fields led to the conclusion of a possible global thermal dissipation. High field Q slope can be encountered in numerous situations, for different cavity frequencies and surface treatments [14-18]. To account for these observations, the mechanism of global thermal limitation in $3.9 \mathrm{GHz}$ cavities, as distinct to lower frequency cavities, appears to be due to the fact that quenches due to local defects occur above the accelerating field that triggers the global thermal instability.

As a first attempt for analytically reconstructing the $\mathrm{Q} v \mathrm{vs}$ $E_{\text {acc }}$ behavior, a simple thermal feedback model was used [19]. The temperature profile inside the cavity can be calculated by finding the equilibrium solution from the heat equation: 


$$
\rho C_{v} \frac{\partial T(\vec{r}, t)}{\partial t}=\nabla \cdot[k \nabla T(\vec{r}, t)]
$$

where $\rho$ is niobium density, $C_{v}$ is niobium specific heat, $k$ is the thermal conductivity. The following boundary conditions were applied to the $\mathrm{Nb}$-vacuum and $\mathrm{He}-\mathrm{Nb}$ interfaces, respectively:

$$
\begin{gathered}
-k\left|\frac{\partial T(z, t)}{\partial z}\right|_{z=0}=\frac{1}{2} R_{s} H_{p}^{2} \\
-k\left|\frac{\partial T(z, t)}{\partial z}\right|_{z=d}=h_{k}\left[T(d, t)-T_{0}\right]
\end{gathered}
$$

where $R_{s}$ is the surface resistance, function of local temperature $T(0, t), H_{p}$ is peak surface magnetic field, $T_{0}$ is the $\mathrm{He}$ bath temperature, $d$ is the wall thickness and $h_{k}$ is Kapitza heat transfer coefficient, which is also a function of the local surface temperature $T(d, t)$. At equilibrium $\frac{\partial T(\vec{r}, t)}{\partial t}=0$, so that Eq. (1) reduces to $\nabla \cdot[k \nabla T(\vec{r}, t)]=0$, which can be solved by simple integration. Assuming small variations in temperature with respect to $T_{0}$, thermal conductivity and the Kapitza heat transfer coefficient can be approximated by their value at $T_{0}$. The cavity heat balance can be expressed by:

$$
\frac{1}{2} R_{s}(T) H^{2}=h\left(T_{0}\right)\left(T-T_{0}\right)
$$

where $\frac{1}{h}=\frac{1}{h_{K}}+\frac{d}{k}$ is a thermal transport parameter-which is temperature dependent-and incorporates the combined effect of heat transfer in the lattice and interface heat transfer through the Kapitza mechanism. Equation (4) allows the evaluation of $\mathrm{rf}$ layer temperature $T$ as a function of surface magnetic field $H$.

Starting from this model we performed an analytical reconstruction of the $\mathrm{Q}$ slope. Surface resistance was expressed as $R_{s}\left(T_{0}\right)=R_{0}+R_{\mathrm{BCS}}\left(T_{0}\right)$, where $R_{\mathrm{BCS}}(T)$ was calculated using the classical quasi-exponential formula with a field-independent band-gap. Temperature dependent thermal conductivity was calculated with the analytic expression presented by Koechlin and Bonin [20], using $\mathrm{RRR}=300$ and phonon mean free path $l=0.1 \mathrm{~mm}$. Several classical references for Kapitza heat transfer coefficient were employed. Here we report only the closest match so far obtained, with the Kapitza parameters coming from measurements on RRR 178 etched niobium samples [21]. Figure 8 shows the result for $3 \mathrm{HZO15}$ at $2 \mathrm{~K}$ and $1.8 \mathrm{~K}$.

It is evident that the thermal feedback model alone is not sufficient to fully reconstruct the Q slope of $3.9 \mathrm{GHz}$ cavities. The large increase of surface resistance, more than doubling this value at $20 \mathrm{MV} / \mathrm{m}$, is not achievable by ordinary heating of the rf surface. Moreover, according to this model the Q values start to decrease at low fields,

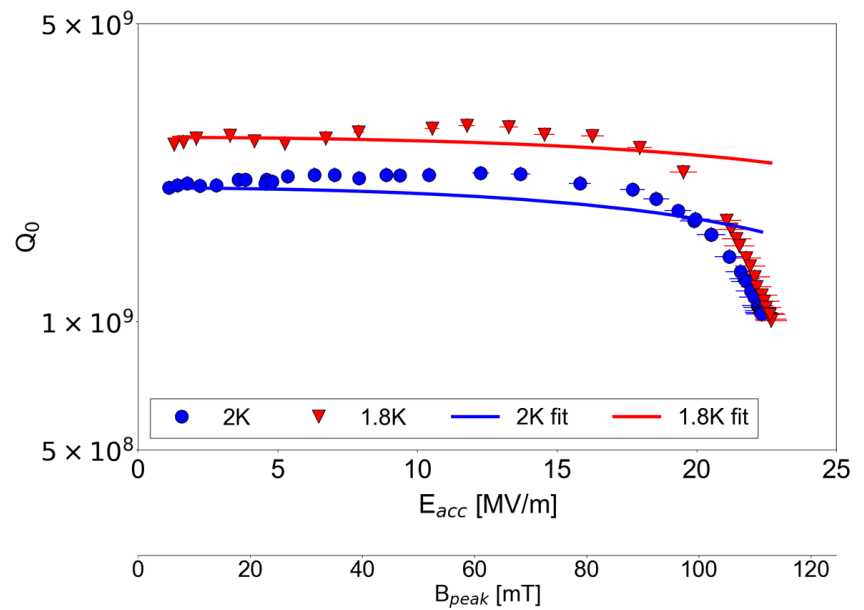

FIG. 8. Experimental and reconstructed Q vs $E_{\text {acc }}$ curves for $3 \mathrm{HZ} 015$ at $2 \mathrm{~K}$ and $1.8 \mathrm{~K}$.

whereas experimental Q-values were stable until a field of approximately $17 \mathrm{MV} / \mathrm{m}$ was reached.

\section{B. The field-dependent BCS resistance model}

We now consider the theory of dissipative nonlinear conductivity under strong magnetic fields, developed by Gurevich [22]. This introduces a nonlinear magnetic field dependence on surface resistance. The classical MattisBardeen theory [23], which describes the response of a low temperature $T<T_{c}$ superconductor to a electromagnetic field, is generalized to the case of high fields, by taking into account the non-equilibrium behavior of quasiparticles. After being thermally excited, quasiparticles interact with the rf field of frequency $\omega$ through the absorption of energy quanta $E=\hbar \omega$. The quasiparticles are driven out of thermodynamic equilibrium by the presence of the screening currents, which broaden the density of states of the quasiparticles and reduce the band gap. A field-dependent quasiparticle gap $\epsilon_{g}(B)<\Delta_{0}$ is therefore defined as the maximum energy at which $N\left(\epsilon_{g}\right)=0$. The strong rf field $B(t)=B_{0} \cos (\omega t)$ produces a periodic smearing of quasiparticle energy gap from $\epsilon=\Delta$ when $B=0$ to $\epsilon=\epsilon_{g}$ when $B=B_{0}$. As a consequence, surface conductivity is time-dependent. A new expression for nonlinear surface conductivity can be obtained by time averaging the rf cycle:

$$
\sigma_{1}(B)=\frac{2 \sigma_{n}}{\pi \hbar} \int_{0}^{\frac{\pi}{\omega}} d t \int_{\epsilon_{g}(t)}^{\infty}[f(\epsilon, s)-f(\epsilon+\hbar \omega, s)] M d \epsilon
$$

where $\sigma_{n}$ is normal state surface conductivity, $f(\epsilon, s)$ is the energy distribution of quasiparticles, $s=s(x, t)=$ $\Delta_{0}\left[\frac{B(t) e^{-x / \lambda}}{2 B_{c}}\right]^{2}$ is the screening current at a depth $\mathrm{x}$ from the $\mathrm{rf}$ surface, $\lambda$ the London penetration depth, $B_{c}$ the thermodynamic critical field, and $M(\epsilon, \omega, B)$ is a spectral function. Surface resistance is then evaluated as: 


$$
R_{s}(B)=\frac{\mu_{0}^{2} \omega^{2} \lambda^{3}}{2 \beta_{0}} \int_{0}^{\beta_{0}} \sigma_{1}(\beta) d \beta
$$

where $\beta_{0}=\left[\frac{B_{0}}{2 B_{c}}\right]^{2}$.

In this mode, the current induced broadening of quasiparticle density of states produces a significant decrease in surface conductivity. This is the favorable side of the nonequilibrium, reducing the value of surface resistance with respect to the predictions of the Mattis-Bardeen theory. Such a mechanism has been employed to explain the anti-Q slope behavior of nitrogen-doped cavities $[24,25]$. On the other hand, the function $f(\epsilon, s)$ in Eq. (5) is different from Fermi-Dirac distribution, which assumes the thermodynamical equilibrium: conversely, it is a complex nonequilibrium distribution accounting for the kinetic balance of the rf power absorption, the recombination of quasiparticles into Cooper pairs and the inelastic scattering of quasiparticles with phonons of niobium lattice. However, typical times for inelastic quasiparticle-phonon scattering and Cooper pair recombination at $2 \mathrm{~K}$ for niobium are $\tau_{s}=$ $1.7 \times 10^{-8} \mathrm{~s}$ and $\tau_{r} \approx 0.4 \times 10^{-6} \mathrm{~s}$, respectively, which are two orders of magnitude greater than the characteristic rf period; that for $3.9 \mathrm{GHz}$ frequency is $2.56 \times 10^{-10} \mathrm{~s}$. This means that quasiparticles do not have enough time to exchange energy with the lattice and change their density during the rf cycle. Hence the quasiparticle temperature, which is higher than the lattice temperature, remains stable during the rf cycle and a static energy distribution can be analytically deduced as function of quasiparticle temperature $f(\epsilon, T)$. This effect, known as quasiparticle overheating, represents the adverse effect of the nonequilibrium behavior. This leads to quasiparticles temperature higher than lattice temperature. Thus, a new term accounting for heat transfer between overheating quasiparticles and lattice phonons has to be considered in the whole cavity heat balance. Thus:

$$
\begin{gathered}
\frac{1}{2} R_{s}(H, T) H^{2}=Y\left(T-T_{i}\right) \\
Y\left(T-T_{i}\right)=h\left(T_{i}-T_{0}\right)
\end{gathered}
$$

where $R_{s}(H, T)=R_{0}+R_{\mathrm{BCS}}(H, T)$, with field dependent BCS resistance expressed by Eq. (6), $T$ is quasiparticle temperature, $T_{i}$ the lattice temperature, $h$ is the thermal coefficient already defined in Eq. (4), and $Y$ is a term quantifying the quasiparticle-phonon transfer rate. Eventually, assuming that $T-T_{0} \ll T_{0}$ the set in Eq. (7) reduces to:

$$
\frac{1}{2} R_{s}(H, T) H^{2}=\frac{1}{\alpha^{\prime}}\left(T-T_{0}\right)
$$

where the parameter $\alpha^{\prime}=\frac{1}{Y}+\frac{1}{h}=\frac{1}{Y}+\frac{1}{h_{K}}+\frac{d}{k}$, known as the normalized overheating parameter, encloses all material parameters: the quasiparticle-phonon energy transfer through $Y$, the heat conduction in the bulk through the thermal conductivity $k$, and the heat transfer in the $\mathrm{Nb}-\mathrm{He}$ interface through the Kapitza mechanism $h_{K}$. Together with Eqs. (6), (9) allows the calculation of the quasiparticle temperature and the determination of a self-consistent fielddependent surface resistance.

A more in-depth theoretical treatment of the fielddependent BCS resistance is found in [22], where the analytical expressions of all the above mentioned parameters, such as quasiparticle distribution, field-dependent energy gap and spectral function, are rigorously defined. The same model was used here to analyze the behavior of $3.9 \mathrm{GHz}$ cavities at high field. Such an approach applies mainly to dirty superconductors which is not truly our case, but we assume the cavity rf surface is nearer to the dirty limit $\left(l_{e}<\xi_{0}\right)$ than to clean limit $\left(l_{e} \gg \xi_{0}\right)$ since, as reported in [2], $l_{e} \sim \xi_{0}$ for most of the cavities.

The role of the overheating parameter is crucial for the quantitative evaluation of surface resistance as a function of accelerating field. Unfortunately no simple analytical expression, nor experimental measurement, is available at high magnetic fields for the quasiparticle-phonon energy transfer coefficient $Y$, as its value is also influenced by the effect of local inhomogeneities and impurities. For this reason, $\alpha^{\prime}$ is regarded in practical calculations as a free parameter. Thus, the $Q$ vs $E_{\text {acc }}$ experimental curve is compared with the reconstructed $Q\left(E_{\text {acc }}, \alpha\right)$ and the closest match is chosen as the true value for the overheating parameter.

If global thermal breakdown is assumed to be the limiting mechanism, the breakdown field, $H_{b}$, defined as the highest magnetic field for which Eq. (9) has a solution as a function of $T$, provides an additional constraint. Figure 9 shows the two terms of Eq. (9) as function of quasiparticle temperature, in the three cases $H<H_{b}$, $H=H_{b}$ and $H>H_{b}$.

For $H<H_{b}$, the solution is the intersection of the line $\frac{1}{\alpha^{\prime}}\left(T-T_{0}\right)$ with the dissipated power term $\frac{1}{2} R_{s}(H, T) H^{2}$ expressed as function of temperature. For $H>H_{b}$, the dissipated power overwhelms the heat transport term and the system is thermally unstable. For $H=H_{b}$, the two curves are tangent for $T_{\max }=T_{e q}\left(H_{b}\right)$. This means that not only the two terms in Eq. (9) are equal, but also their temperature derivatives:

$$
\left.\frac{1}{2} \frac{d R_{s}\left(H_{b}, T\right)}{d T}\right|_{T_{\max }} H_{b}^{2}=\frac{1}{\alpha^{\prime}}
$$

Substituting $\alpha^{\prime}$ in Eq. (9) and rearranging, one obtains:

$$
R_{s}\left(H_{b}, T_{\max }\right)=\left.\frac{d R_{s}\left(H_{b}, T\right)}{d T}\right|_{T_{\max }}\left(T_{\max }-T_{0}\right)
$$

which allows calculation of $T_{\max }$, and then from Eq. (9) the overheating parameter $\alpha^{\prime}$. 


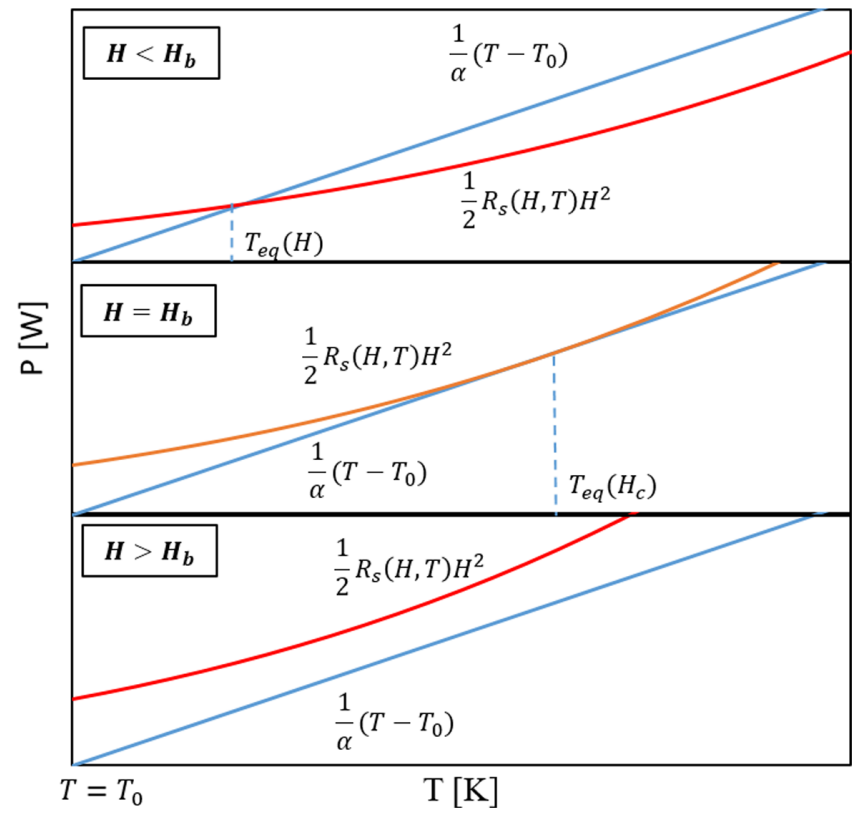

FIG. 9. Dissipated power and heat transport term versus quasiparticle temperature, in the three cases $H<H_{b}, H=H_{b}$, and $H>H_{b}$. Equilibrium temperature is at the intersection of the two curves.

In summary, if the experimentally measured quench field is considered as the global thermal breakdown gradient limit, one can deduce the overheating parameter from the solution of Eq. (11). It must be stressed, however, that a temperature independent overheating parameter needs to assumed. The accuracy of this method needs to be checked in the case of high quasiparticle overheating.

\section{Simulation results and discussion}

The derivation of the XFEL $3.9 \mathrm{GHz}$ cavities experimental Q vs $E_{\text {acc }}$ curves with the field-dependent surface resistance model was performed as follows: (i) Experimental values for $R_{0}$ and $R_{\mathrm{BCS}}$ at low field were extracted from experimental data. The values used for each cavity were as reported previously [1]. The measured quench field was assumed to be the value triggering the global instability, namely the breakdown field $H_{b}$ as defined in Eq. (10). (ii) Field dependent surface resistance given by Eq. (6) was calculated at $H=H_{b}$ and the function of temperature $R_{s}\left(H_{b}, T\right)$ was derived. $T_{\max }$ and $\alpha^{\prime}$ were experimentally calculated from Eq. (11). (iii) Having calculated the overheating parameter, the surface resistance at different fields was calculated with Eq. (9). The Q value was calculated as $Q_{0}\left(E_{\mathrm{acc}}\right)=\frac{G}{R_{\mathrm{BCS}}\left(E_{\mathrm{acc}}\right)+R_{0}}$, where $G$ is the cavity geometry factor. As $R_{\mathrm{BCS}}$ is the dominant contribution to total surface resistance due to its $f^{2}$ dependence, we neglected any field dependence of the residual resistance term. Figure 10 shows the results of the reconstruction performed for cavity $3 \mathrm{HZ} 015$ at $2 \mathrm{~K}, 1.8 \mathrm{~K}$, and $1.7 \mathrm{~K}$, compared with the

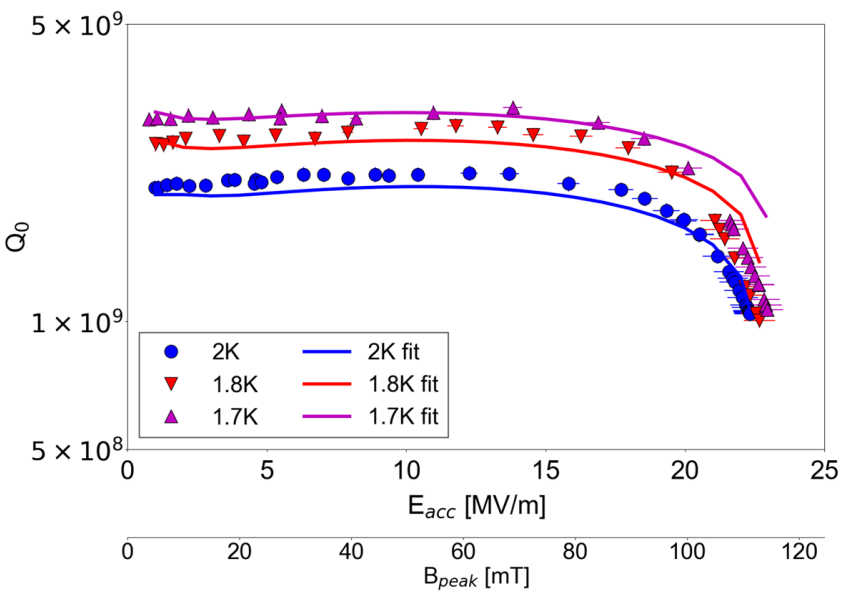

FIG. 10. Comparison between measured and calculated (fit) $Q_{0}$ vs $E_{\text {acc }}$ curves for cavity $3 \mathrm{HZ} 015$ at $2 \mathrm{~K}, 1.8 \mathrm{~K}$, and $1.7 \mathrm{~K}$.

experimental Q vs $E_{\text {acc }}$ curves. A $5 \%$ relative error was assumed for both the experimental values of $Q_{0}$ and $E_{\text {acc }}$ [26]. The error of the calculated $Q_{0}$ was evaluated by means of error propagation assuming $5 \%, 5 \%$, and $10 \%$ as the relative errors for $E_{\text {acc }}$, low field $R_{\mathrm{BCS}}$ and $R_{0}$, respectively. For the sake of legibility, error bars are omitted from the graph.

There was a good agreement of the model results with experimental data at $2 \mathrm{~K}$. The prediction of the model for both the Q slope and Q-value at breakdown field matched well with the corresponding experimental values. For $1.8 \mathrm{~K}$ and $1.7 \mathrm{~K}$, the model underestimated the measured decrease of $Q_{0}$ with increasing accelerating field. The spread between experimental and simulated values is nevertheless noticeably decreased when compared to that of the thermal feedback model.

A slight increase of $\mathrm{Q}$ at medium field was also noticed. Calculated values ranged from $1.9 \times 10^{9}$ at $3 \mathrm{MV} / \mathrm{m}$ to $2.07 \times 10^{9}$ at $15 \mathrm{MV} / \mathrm{m}$, similar to what happens in experimental data. The increase was $5 \%$ for the simulation, slightly less than the experimentally observed $8 \%$ increase. The mechanism accounting for this slight anti-Q slope could be the same that activates the vigorous anti-Q slope of nitrogen doped cavities.

The values of $\alpha^{\prime}$ for cavity 3HZ015 at the different temperatures are reported in Table II. A slight dependence of the overheating parameter on temperature can be noticed, consistent with the results obtained by others [24].

TABLE II. $\quad \alpha^{\prime}$ versus Helium bath temperature.

\begin{tabular}{lc}
\hline \hline He bath temp. $[\mathrm{K}]$ & $\alpha^{\prime}\left[\mathrm{m}^{2} \mathrm{~K} / \mathrm{W}\right]$ \\
\hline 1.7 & 0.52 \\
1.8 & 0.48 \\
2.0 & 0.44 \\
\hline \hline
\end{tabular}


Very similar values were obtained for the other $3.9 \mathrm{GHz}$ series cavities, with some exceptions.

Figure 11 shows the results of the reconstruction for other $3.9 \mathrm{GHz}$ series cavities at $2 \mathrm{~K}$. 3HZ013 and 3HZ017 have critical fields close to that of $3 \mathrm{HZ} 015$, as shown by the calculated $\alpha^{\prime}$. However, cavity $3 \mathrm{HZO0} 4$ quenched at $20 \mathrm{Mv} /$ $\mathrm{m}$ with a full Q-slope behavior. The estimated overheating parameter was higher, although treatment and materials used were the same as in other series cavities. Looking back to the processing experience reported in [1], cavity 3HZ004 was BCP treated at the beginning of the first batch, when

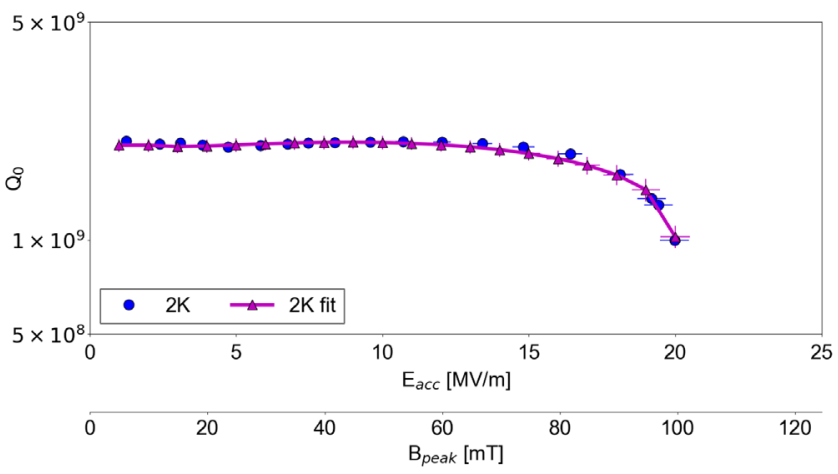

(a) 3HZ004. $\alpha=0.53 \cdot 10^{-3} \frac{m^{2} K}{W}, E_{\max }=20 \frac{M V}{m}$

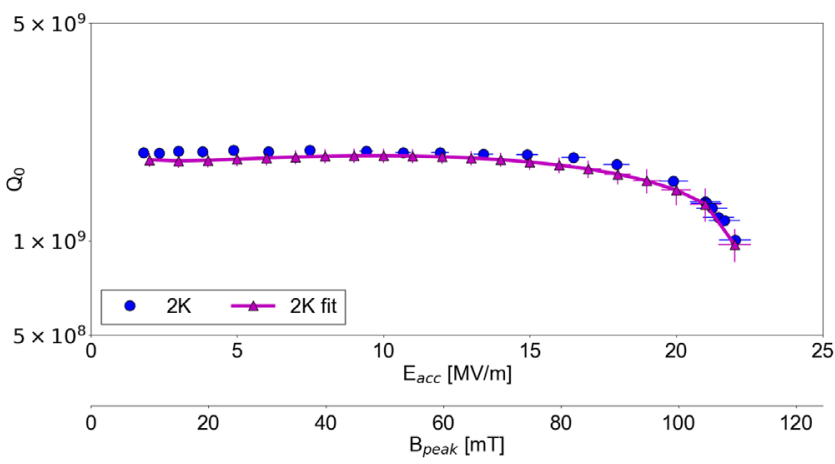

(b) 3HZ013. $\alpha=0.44 \cdot 10^{-3} \frac{m^{2} K}{W}, E_{\max }=22.0 \frac{M V}{m}$

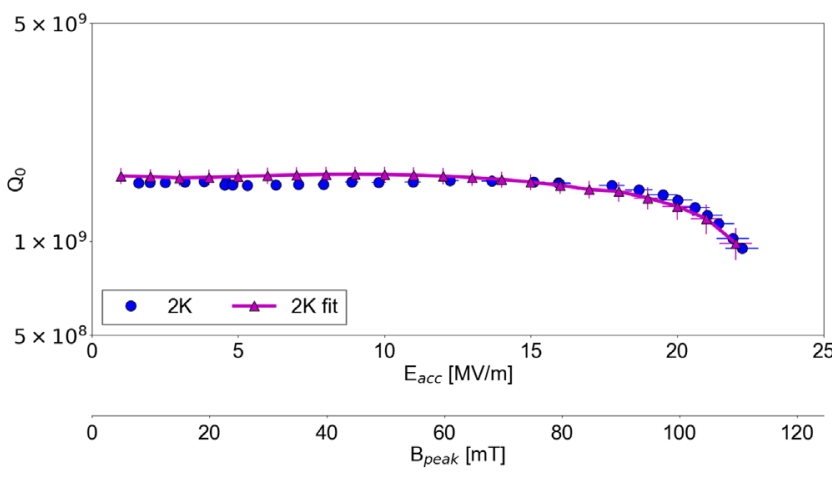

(c) 3HZ017. $\alpha=0.40 \cdot 10^{-3} \frac{m^{2} K}{W}, E_{\max }=22.2 \frac{M V}{m}$

FIG. 11. Comparison between measured and calculated $Q_{0}$ vs $E_{\text {acc }}$ curves for cavity 3HZ004 (a), 3HZ013 (b), and 3HZ017 (c) at $2 \mathrm{~K}$. Errors on calculated value are also indicated. The estimated overheating parameters and the breakdown fields are reported in the subcaption. treatment parameters were still not optimized. For example, the nominal acid flow had not yet been set at $1.5 \mathrm{~L} / \mathrm{min}$. This could explain the slight difference in performance of this cavity and may suggest an influence of final BCP treatment on the high field Q slope behavior.

It is worthwhile to evaluate the weight of each single heat transport contribution to the normalized overheating parameter. Employing the classical values of $k=75 \mathrm{~W} /(\mathrm{mK})$ for thermal conductivity, $h_{K}=1 \times 10^{4} \mathrm{~W} /\left(\mathrm{m}^{2} \mathrm{~K}\right)$ for Kapitza heat transfer coefficient, and $d=2.6 \mathrm{~mm}$ as wall thickness, one obtains $\frac{1}{h}=\frac{1}{h_{K}}+\frac{d}{k} \approx 0.13 \times 10^{-3} \mathrm{~W} /\left(\mathrm{m}^{2} \mathrm{~K}\right)$. Hence, the quasiparticle-phonon energy transfer coefficient can be estimated as $\frac{1}{Y}=\alpha^{\prime}-\frac{1}{h} \approx 0.31 \times 10^{-3} \mathrm{~m}^{2} \mathrm{~K} / \mathrm{W}$. It contributes $70 \%$ to the overheating normalized parameter. This means that the true bottleneck in cavity heat transfer is the quasiparticle-phonon interaction. In fact the temperature of the rf layer is higher due to the low efficiency of quasiparticles in reaching thermodynamic equilibrium with the lattice. The experimentally observed linear dependence of $\frac{1}{Y}$ to electron mean free path suggests a crucial role for impurities in moderating quasiparticle energy. An impurity embedded in the lattice may act as a scattering center for quasiparticles and favor energy transfer with lattice phonons.

The discrepancies observed between experimental and calculated values at lower temperatures deserves some comment. Several assumptions were made in order to apply the field-dependent surface resistance model to the experimental results. Specifically, this involved the linearization of the heat transport term allowing, through Eq. (11), to evaluate the overheating coefficient $\alpha$ as the derivative of $R\left(H_{b}, T\right)$ at the breakdown temperature. Such an approach cannot be used without this assumption. There is a need to know the temperature dependence of $\alpha^{\prime}$ in order to generalize Eq. (11) to the case of temperature-dependent overheating. Within this constraint, linearization offers an initial means of assessing of the limiting performance of cavities at $2 \mathrm{~K}$.

Several other approximations are intrinsic in the fielddependent surface resistance model. These include no simple analytical expression for field dependent band gap existing in the clean limit. The field dependence for band gap takes into account only the quadratic field correction. The nonlinear Meissner effect, introduces a field dependence for London penetration depth. The effect of surface roughness, which is known to be influential on performance limiting mechanisms, should also be carefully considered. The enhancement of surface magnetic field due to the rough BCP-treated surface can locally increase the dissipated power and therefore introduce an additional contribution to the Q-slope [27]. Finally, the residual resistance due to trapped magnetic flux is also field dependent $[28,29]$, and is likely to influence the high-field $\mathrm{Q}$ behavior in interplay with BCS surface resistance. In the case of $3.9 \mathrm{GHz}$, assuming a trapping flux sensitivity of 
$0.5 \mathrm{n} \Omega / \mathrm{mG}$ at low $E_{\text {acc }}$ for a BCP-treated cavity [30], an external field of $10 \mathrm{mG}$ would give an overall contribution of $5 \mathrm{n} \Omega$ to low-field surface resistance. Even assuming a worst case scenario where the trapped flux sensitivity reaches $1.5 \mathrm{n} \Omega / \mathrm{mG}$ at $20 \mathrm{Mv} / \mathrm{m}$, as pointed out in [30], the trapped flux contribution to residual resistance would not be more than $15 \mathrm{n} \Omega$. Hence, the BCS surface resistance-which is of the order of $100 \mathrm{n} \Omega$ - would overwhelm any contribution of trapped magnetic flux, and at $2 \mathrm{~K}$ one can neglect its dependence on magnetic field. Alternatively, at lower temperatures $R_{\mathrm{BCS}}(T)$ decreases, eventually approaching a magnitude equivalent to the residual resistance. This may be a possible explanation of the discrepancies observed in our model at lower temperatures. In the future, the model will be extended to incorporate the most recent theories for the field dependence of sensitivity to trapped flux.

\section{CONCLUSIONS}

The results of vertical cold tests of the $3.9 \mathrm{GHz}$ superconducting cavities for the European XFEL were analyzed and a mechanism to explain the limits in cavity performance has been proposed.

A global thermal dissipation mechanism was identified, accounting for limiting of performance at approximately $22 \mathrm{Mv} / \mathrm{m}$. Pivotal was the triggering of a high field Q slope starting from $17 \mathrm{Mv} / \mathrm{m}$, induced by the high surface resistance (because of the $f^{2}$ dependence of $R_{\mathrm{BCS}}(T)$ ). The highfield behavior was quantitatively reconstructed by only considering nonequilibrium superconductivity. High frequency plays a significant role in the overheating of the rf surface due to the kinetic balance between the short rf period of quasiparticle recombination and quasiparticle-phonon scattering. The fit results matched the experimental data at $2 \mathrm{~K}$. The discrepancies at lower temperatures could be due to the field dependence of residual resistance, which was not included. An upgrade of the mathematical model taking into account this contribution will be undertaken in the future.

Performance of the cavities clearly is dependent on surface treatment, which in this case was based on BCP. The $\mathrm{BCP}$ recipe employed is not optimal for achieving stateof-the-art performances but it was motivated by the need to meet the moderate specification value of $15 \mathrm{Mv} / \mathrm{m}$ for the EXFEL third harmonic cavities. One would expect different breakdown fields and Q-slope onsets with different treatments. These could include a low temperature baking and using electropolishing bulk treatment with nitrogen doping. In the future, we plan to use these alternative treatments on $3.9 \mathrm{GHz}$ cavities to investigate the influence of the surface preparation on the field-dependent surface resistance.

[1] P. Pierini, M. Bertucci, A. Bosotti, J. Chen, C. G. Maiano, P. Michelato, L. Monaco, M. Moretti, P. Pagani, R. Paparella, D. Sertore, and E. Vogel, Fabrication and vertical test experience of the European X-ray Free Electron Laser $3.9 \mathrm{GHz}$ superconducting cavities, Phys. Rev. Accel. Beams 20, 042006 (2017).

[2] M. Bertucci, A. Bignami, A. Bosotti, J. Chen, C. Maiano, P. Michelato, L. Monaco, R. Paparella, P. Pierini, D. Sertore, and C. Pagani, in Proc. of the ninth Particle Accelerator Conference (IPAC'18), Vancouver, BC, Canada, April 29-May 4, 2018, International Particle Accelerator Conference No. 9, JACoW (JACoW publishing, Geneva, Switzerland, 2018) p. 2644.

[3] E. Vogel et al., in Proc. of International Particle Accelerator Conference (IPAC'10), Kyoto, Japan, May 23-28, 2010, International Particle Accelerator Conference No. 1, JACoW (JACoW publishing, Geneva, Switzerland, 2010) pp. 4281-4283.

[4] G. Ciovati, SUPERFIT: A computer code to fit surface resistance and penetration depth of a superconductor, JLAB Technical Report No. TN 03-003, 2003.

[5] H. Padamsee, J. Knobloch, and T. Hays, RF Superconductivity for Accelerators, Wiley Series in Beam Physics and Accelerator Technology (Wiley, New York, 1998), p. 88.

[6] B. Bonin, in Proceedings of Cern Accelerator SchoolSuperconductivity in Particle AcceleratorsHaus Rissen, Hamburg, Germany, 1995, 96-03 (CERN, Geneva, 1996), p. 195.

[7] A. Romanenko and L. V. Goncharova, in Proc. of 15th International Conference on $R F$ Superconductivity (SRF '11), Chicago, Illinois, USA, July 25-29, 2011, International Conference on RF Superconductivity No. 15, JACoW (JACoW publishing, Geneva, Switzerland, 2012), p. 849.

[8] M. Martinello, M. Checchin, A. Romanenko, A. Grassellino, S. Aderhold, S. K. Chandrasekaran, O. Melnychuk, S. Posen, and D. A. Sergatskov, Field-Enhanced Superconductivity in High-Frequency Niobium Accelerating Cavities, Phys. Rev. Lett. 121, 224801 (2018).

[9] M. Ge, G. Hoffstaetter, F. Furuta, E. Smith, M. Liepe, S. Posen, H. Padamsee, D. Hartill, and X. Mi, in Proc. of North American Particle Accelerator Conference (NA-PAC '13), Pasadena, CA, USA, Sep. 29-Oct. 4, 2013, North American Particle Accelerator Conference No. 2, JACoW (JACoW publishing, Geneva, Switzerland, 2014), p. 805.

[10] R. A. Sherlock and D. O. Edwards, Oscillating superleak second sound transducers, Rev. Sci. Instrum. 41, 1603 (1970).

[11] P. Zhang and M. Murakami, Three dimensionality of pulsed second-sound waves in He II, Phys. Rev. B 74, 024528 (2006).

[12] M. Bertucci, A. Bosotti, L. Garolfi, P. Michelato, L. Monaco, D. Sertore, and C. Pagani, in Proc. of 16th International Conference on RF Superconductivity (SRF '13), Paris, France, Sept. 23-27, 2013, International Conference on RF Superconductivity No. 16, JACoW (JACoW publishing, Geneva, Switzerland, 2014) p. 710.

[13] E. Harms, H. Edwards, A. Hocker, T. Khabiboulline, and N. Solyak, in Proc. of the 24th Linear Accelerator Conference (LINAC '08), Victoria, British Columbia, Canada, Sept. 29-Oct. 3, 2008, International Conference on Linear Accelerator No. 24, JACoW (JACoW publishing, Geneva, Switzerland, 2008), pp. 845-847. 
[14] J. Vines, Y. Xie, and H. Padamsee, in Proc. of 13th International Conference on $R F$ Superconductivity (SRF2007), Bejing, China, Oct. 14-19, 2007, International Conference on RF Superconductivity No. 24, JACoW (JACoW publishing, Geneva, Switzerland, 2007), p. 178.

[15] G. Ciovati and J. Halbritter, Analysis of the medium field Q-slope in superconducting cavities made of bulk niobium, Physica C 441, 57 (2006).

[16] L. Lilje, D.Reschke, K. Twarowsky, P. Schmüser, D. Bloess, E. Haebel, E. Chiaveri, J. M. Tessier, H. Preis, H. Wenninger, H. Safa, and J. P. Charrier, in Proc. of International Conference on $R F$ Superconductivity (SRF1999), Santa Fe, NM, USA, Nov. 1-5, 1999, International Conference on RF Superconductivity No. 9, JACoW (JACoW publishing, Geneva, Switzerland, 1999), p. 74.

[17] G. Ciovati, G. Eremeev, and F. Hannon, High field $Q$ slope and the effect of low-temperature baking at $3 \mathrm{GHz}$, Phys. Rev. Accel. Beams 21, 012002 (2018).

[18] P. Dhakal, G. Ciovati, and G. R. Myneni, Role of thermal resistance on the performance of superconducting radio frequency cavities, Phys. Rev. Accel. Beams 20, 032003 (2017).

[19] A. Gurevich, Physica C 441, 38 (2006).

[20] F. Koechlin and B. Bonin, Parametrization of the niobium thermal conductivity in the superconducting state, Supercond. Sci. Technol. 9, 453 (1996).

[21] H. Edwards, C. A. Cooper, M. Ge, I. V. Gonin, E. R. Harms, T. N. Khabiboulline, and N. Solyak, in Proc. 14th International Conference on RF Superconductivity (SRF '09), Berlin, Germany, September 20-25, 2009, International Conference on RF Superconductivity No. 14, JACoW (JACoW publishing, Geneva, Switzerland, 2009), p. 379.

[22] A. Gurevich, Reduction of Dissipative Nonlinear Conductivity of Superconductors by Static and Microwave Magnetic Fields, Phys. Rev. Lett. 113, 087001 (2014).
[23] D. C. Mattis and J. Bardeen, Theory of the anomalous skin effect in normal and superconducting metals, Phys. Rev. 111, 412 (1958).

[24] J. T. Maniscalco, D. Gonnella, and M. Liepe, The importance of the electron mean free path for superconducting radio-frequency cavities, J. Appl. Phys. 121, 043910 (2017).

[25] M. Martinello, S. Aderhold, S. K. Chandrasekaran, M. Checchin, A. Grassellino, O. Melnychuk, S. Posen, A. Romanenko, and D. Sergatskov, in Proceedings of the 18th International Conference on RF Superconductivity (SRF2017), Lanzhou, China, July 17-21, 2017, International Conference on RF Superconductivity No. 18, JACoW (JACoW publishing, Geneva, Switzerland, 2018), p. 364.

[26] O. Melnychuk, A. Grassellino, and A. Romanenko, Error analysis for intrinsic quality factor measurement in superconducting radio frequency resonators, Rev. Sci. Instrum. 85, 124705 (2014).

[27] C. Xu, C.E. Reece, and M. J. Kelley, Simulation of nonlinear superconducting rf losses derived from characteristic topography of etched and electropolished niobium surfaces, Phys. Rev. Accel. Beams 19, 033501 (2016).

[28] A. Romanenko and A. Grassellino, Dependence of the microwave surface resistance of superconducting niobium on the magnitude of the rf field, Appl. Phys. Lett. 102, 252603 (2013).

[29] C. Benvenuti, S. Calatroni, I. Campisi, P. Darriulat, M. A. Peck, R. Russo, and A.-M. Valente, Study of the surface resistance of superconducting niobium films at $1.5 \mathrm{GHz}$, Physica C 316, 153 (1999).

[30] M. Checchin, M. Martinello, A. Grassellino, S. Aderhold, K. Chandrasekaran, O. Melnychuk, S. Posen, A. Romanenko, and D. A. Sergatskov, Frequency dependence of trapped flux sensitivity in SRF cavities, Appl. Phys. Lett. 112, 072601 (2018). 